\title{
Selection of the high efficient sgRNA for CRISPR-Cas9 to edit herbicide related genes, PDS, ALS, and EPSPS in tomato
}

\author{
So Hee Yang, Euyeon Kim, Hyosun Park and Yeonjong Koo*
}

\begin{abstract}
Herbicide resistance is one of the main crop traits that improve farming methods and crop productivity. CRISPR-Cas9 can be applied to the development of herbicide-resistant crops based on a target site resistance mechanism, by editing genes encoding herbicide binding proteins. The sgRNAs capable of editing the target genes of herbicides, pds (phytoene desaturase), ALS (acetolactate synthase), and EPSPS (5-Enolpyruvylshikimate-3-phosphate synthase), were designed to use with the CRISPR-Cas9 system in tomato (Solanum lycopersicum cv. Micro-Tom). The efficiency of the sgRNAs was tested using Agrobacterium mediated transient expression in the tomato cotyledons. One sgRNA designed for editing the target site of PDS had no significant editing efficiency. However, three different sgRNAs designed for editing the target site of ALS had significant efficiency, and one of them, ALS2-P sgRNA, showed over $0.8 \%$ average efficiency in the cotyledon genome. The maximum efficiency of ALS2-P sgRNA was around 1.3\%. An sgRNA for editing the target site of EPSPS had around $0.4 \%$ editing efficiency on average. The sgRNA efficiency testing provided confidence that editing of the target sites could be achieved in the transformation process. We confirmed that 19 independent transgenic tomatoes were successfully edited by ALS2_P or ALS1_W sgRNAs and two of them had three base deletion mutations, which are expected to have altered herbicide resistance. In this study, we demonstrated the usefulness of performing an sgRNA efficiency test before crop transformation, and confirmed that the CRISPR-Cas9 system is a valuable tool for breeding herbicide-resistant crops.
\end{abstract}

Keywords: Herbicide resistance, Tomato, CRISPR-Cas9, Acetolactate synthase

\section{Introduction}

Weed outbreaks are the biggest problem facing agriculture worldwide [1,2]. Of the 200,000 plant species on the planet, about $1 \%$ are cultivated crops, and about $0.1 \%$ are considered to be weeds. Weeds reduce crop productivity by competing with crops for soil moisture, nutrients, $\mathrm{CO}_{2}$, light, and space. Weed growth is one of the major factors affecting crop quality and yield worldwide [3]. Many methods have been used to remove problem weeds $[4,5]$. The use of herbicides is the main method of weed control in modern crop production systems [6,

\footnotetext{
*Correspondence: yeonjong@jnu.ac.kr

Department of Agricultural Chemistry, Chonnam National University, Gwangju 61186, Republic of Korea
}

7]. Herbicides that inhibit amino acid synthesis, photosynthesis-related chlorophyll synthesis, or promote ROS production have been developed [4]. In general, nonselective herbicides such as glyphosate do not differentiate between crops and weeds, and cause most plants to die. When glyphosate is applied to plants, it affects the primary point of action of 5-Enolpyruvylshikimate3-phosphate synthase (EPSPS) in the amino acid biosynthesis system, inhibiting the biosynthesis of aromatic amino acids such as tryptophan, tyrosine, and phenylalanine $[8,9]$. The biosynthesis of aromatic amino acids is important to plants, and when it is inhibited, secondary and tertiary changes occur in the body, and the plant dies [10,11]. Therefore, weeds can be controlled by inhibiting the synthesis of aromatic amino acids [7, 
12]. Acetolactate synthase (ALS) is an enzyme essential for the synthesis of branched aliphatic amino acids such as leucine, valine, and isoleucine [13, 14]. Biosynthetic metabolism involving ALS is regulated by the allosteric binding of valine, leucine, and isoleucine to ALS [15]. The ALS inhibitory herbicides imidazolinone and sulfonylurea do not compete with pyruvate [16]. Phytoene desaturase (PDS) is an enzyme that converts phytoene into lycopene located in the carotenoid biosynthesis pathway [17]. The activity of PDS is usually inhibited by herbicides such as norflurazone and fluridone [18]. Carotenoids are known to play an important role in the formation of Photosystem II by contributing to the binding and stability of the protein complex constituting Photosystem II [19]. Herbicides acting through PDS inhibition cause necrosis due to chlorophyll destruction due to carotenoid deficiency in growing leaves [20]. The reduction of carotenoid content induced by PDS inhibition in plant leaves exerts different growth inhibitory effects according to the stage of the leaf growth cycle [21]. As the use of herbicides increased, indiscriminate spraying caused ecological destruction and disturbance, and led to the rapid evolution of herbicide-resistant weeds [22]. Therefore, the development of herbicide-resistant crops using new technologies is important [23, 24].

Most high efficiency herbicide-tolerant crops have been developed by the introduction of target site resistance in genes encoding the target proteins of herbicides, or by non-target site resistance, including herbicide absorption or metabolism. Currently, most commercial herbicidetolerant crops have been obtained by transgenic breeding [25]. The increase in the yields of genetically modified herbicide-tolerant crops is primarily due to the development of glyphosate-resistant soybeans, corn, rice, wheat, and canola [26-28]. Other herbicides, such as ALS inhibitory herbicides, can also improve crop tolerance to herbicides through transgenic breeding techniques.

An alternative approach to crop development involves the use of gene editing, which has recently been spotlighted $[29,30]$. Gene editing is a technique used to edit DNA [31, 32]. The gene editing process involves first-generation Zinc Finger Nucleases (ZFNs), secondgeneration Transcription Activator-Like Effector Nucleases (TALENs), and third-generation clustered regular interspaced short palindromic repeats (CRISPR) [33]. The third-generation gene editing method involves the use of the CRISPR Cas9 system is a system to edit genes at a specific location. Compared to other gene editing methods such as ZFN and TALLEN, it can detect RNA-derived target DNA binding domains, making the production process easy and low cost. It is an innovative system used by many researchers because of its accuracy and simplicity $[34,35]$. The guide RNA (gRNA) used as the target gene of the CRISPR system serves to impart specificity of the target sequence to the CRISPRCas9 system [33, 36]. The process of gene editing, which used to take months to years to cut and replace a single gene, was shortened to a few days with the development of CRISPR, and this system led to breakthroughs in gene editing, such as the ability to edit multiple genes simultaneously [30]. The production of herbicide-resistant crops using the CRISPR Cas9 system appears to be the preferred method of weed control to date [37]. Herbicidetolerant crops have the advantage of reducing toxicity to the human body and the environment by at least threefold, by reducing the amount of herbicide used compared to conventional crop cultivation. It increases crop yield by removing only problem weeds without damaging crops [38]. It is considered to be an essential factor for large-scale cultivation, labor reduction, and cost reduction, because it increases the efficiency of weed control. In order to utilize gene editing, a method of inducing mutation of an endogenous gene may be used. By analyzing the amino acid sequence of target proteins, gRNA can be designed so that gene editing occurs at the site where the substrate binds, and crops with low sensitivity to herbicides can be selected from the range of edited crops produced. In this study, a guide RNA for target gene selection was designed using the CRISPR Cas9 system, for the production of herbicide-resistant crops. Based on the herbicide-resistant protein studies published to date, the efficiency of various guide RNAs for target proteins and target amino acids was compared for the development of highly efficient herbicide-resistant tomatoes.

\section{Results and discussion Design of sgRNA}

The genes involved in the response to the three most commonly used herbicides, norflurazone, sulfonylurea/imidazolinone, and glyphosate, are pds1 (phytoene desaturase), ALS (acetolactate synthase), and EPSPS (5-Enolpyruvylshikimate-3-phosphate synthase), respectively. One $p d s 1$ gene (Solyc03g123760), two different $A L S$ genes (Solyc06g059880 and Solyc03g044330), and one EPSPS gene (Solyc01g091190) have been identified in the tomato genome (Solanum lycopersicum). The target amino acids which are expected to bind substrates are marked with corresponding genome sequences (Fig. 1). According to an amino acid sequence alignment, the target amino acids are conserved in various plants, including Arabidopsis. The Proto-Spacer Adjacent Motif (PAM) 5'-NGG-3' sequence was selected near the target sites, and $20 \mathrm{nt}$ of sequence upstream of the PAM sequence was selected as the sgRNA. In the case of the $p d s 1$ and EPSPS genes, sgRNA was additionally selected at other positions in each gene (PDS-c, EPSPS-c1, EPSPS-c2), to 


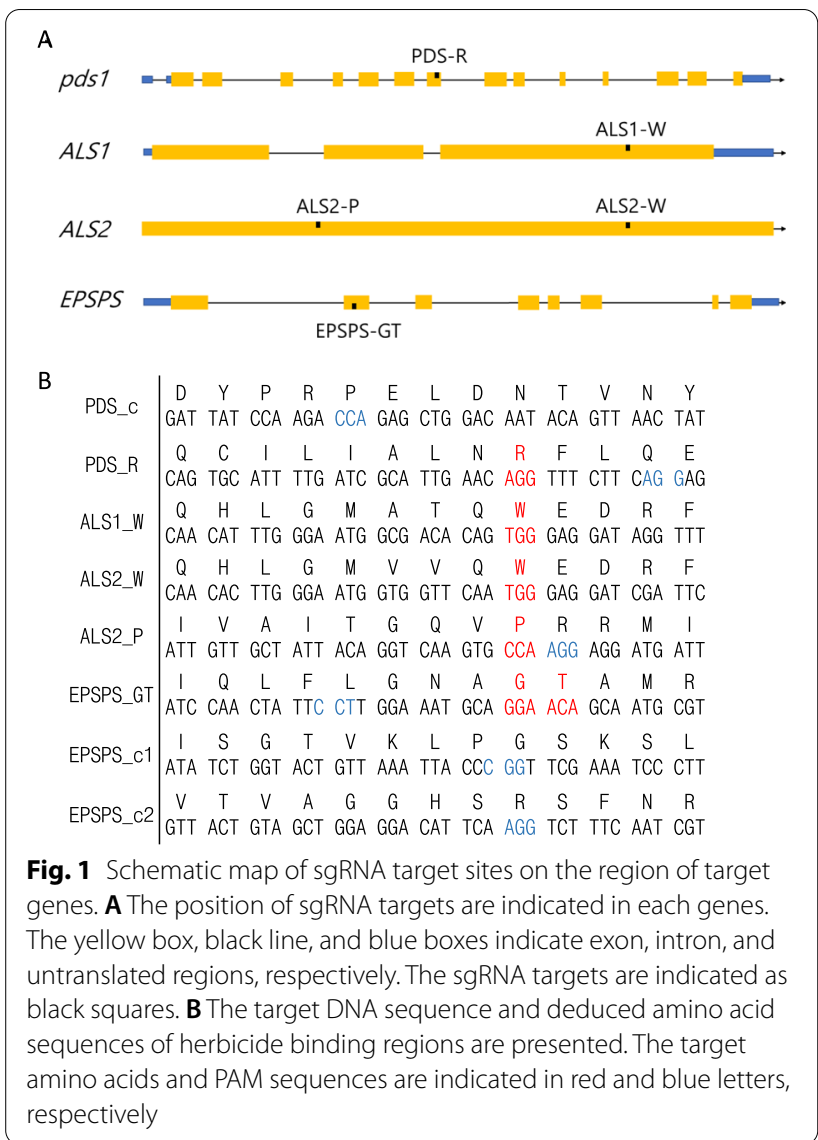

facilitate comparison of sgRNA editing efficiency. Table 1 shows the selected sgRNA sequences and the estimated efficiency calculated from the CRISPR-P 2.0 program for each sgRNA sequence. According to this estimation, PDS-D sgRNA was not efficient compared to the control sgRNA, PDS-c. ALS1-W and ALS2-P were expected to have high editing efficiency compared to the ALS2W sgRNA. The EPSPS-GT sgRNA has higher efficiency compared to the two different control sgRNAs. Therefore, the three sgRNAs of the ALS1, ALS2, and EPSPS genes were designed to produce herbicide-resistant proteins edited using the CRISPR-Cas9 system.

\section{CRISPR-Cas9 vector construction}

In the CRISPR-Cas9 system we used, the sgRNA is transcribed by the Arabidopsis $\mathrm{U} 6$ promoter $\left(\mathrm{P}_{\mathrm{U} 6}\right)$ and the Cas9 gene is expressed ectopically by the $35 \mathrm{~S}$ promoter (Fig. 2). An Arabidopsis codon optimized Cas9 protein is used in this system [39]. The backbone of the destination vector is the pMDC32 vector, which has a kanamycin resistance gene as a bacterial selection marker and a hygromycin resistance gene as a plant selection marker. The tomato Micro-Tom cultivars were successfully selected by hygromycin on the selection media under the conditions described in the methods section.

\section{Transient expression tests identified efficient sgRNAs}

Efficiency testing of sgRNA was performed by injecting Agrobacterium into tomato cotyledons and analyzing the genomes of the cotyledons. The target gene site was amplified using the primers shown in Table 2, and amplified fragments were analyzed using next-generation sequencing (NGS). In this study, the number of base deletions and insertions at the target site were counted to calculate the editing efficiency. We statistically analyzed the results of at least six replicates for each sgRNA.

The median value of the target gene PDS showed efficiencies for PDS-c of 0.06 and for PDS-R of 0.014 (Fig. 3A). These values were not significant as the editing efficiency of sgRNA, because they are lower than the chance of misreading in NGS, which is around $0.1 \%$ of total readings. In the case of sgRNA PDS-c, the experimental value was not significant, but it was predicted to able to efficiently edit targets according to the analysis produced by the CRISPR-P 2.0 program, which calculated a value of 0.2317 (Fig. 3A and Table 1). These results indicate that the location of the pds1 gene is poorly accessible to the CRISPR-Cas9 complex.

Table 1 Selected sgRNA for targeting herbicide resistance genes

\begin{tabular}{llll}
\hline Gene full name & Target gene name & sgRNA sequence & On-score* \\
\hline Phytoene desaturase1 & PDS_C & TTAACTGTATTGTCCAGCTC & 0.2317 \\
Phytoene desaturase2 & PDS_R & CGCATTGAACAGGTTTCTTC & 0.0187 \\
Acetolactate synthase 1 & ALS1_W & ATTTGGGAATGGCGACACAG & 0.3573 \\
Acetolactate synthase 2 & ALS2_W & ACTTGGGAATGGTGGTTCAA & 0.0626 \\
& ALS2_P & CTATTACAGGTCAAGTGCCA & 0.2895 \\
5-Enolpyruvylshikimate-3-phosphate & EPSPS_GT & TTGCTGTTCCTGCATTTCCA & 0.3950 \\
synthase & EPSPS_C1 & TCTGGTACTGTTAATTACC & 0.4788 \\
& EPSPS_C2 & CTGTAGCTGGAGGACATTCA & 0.0776 \\
\hline
\end{tabular}

* sgRNA target score estimated using the CRISPR-P 2.0 program. http://crispr.hzau.edu.cn/CRISPR2 


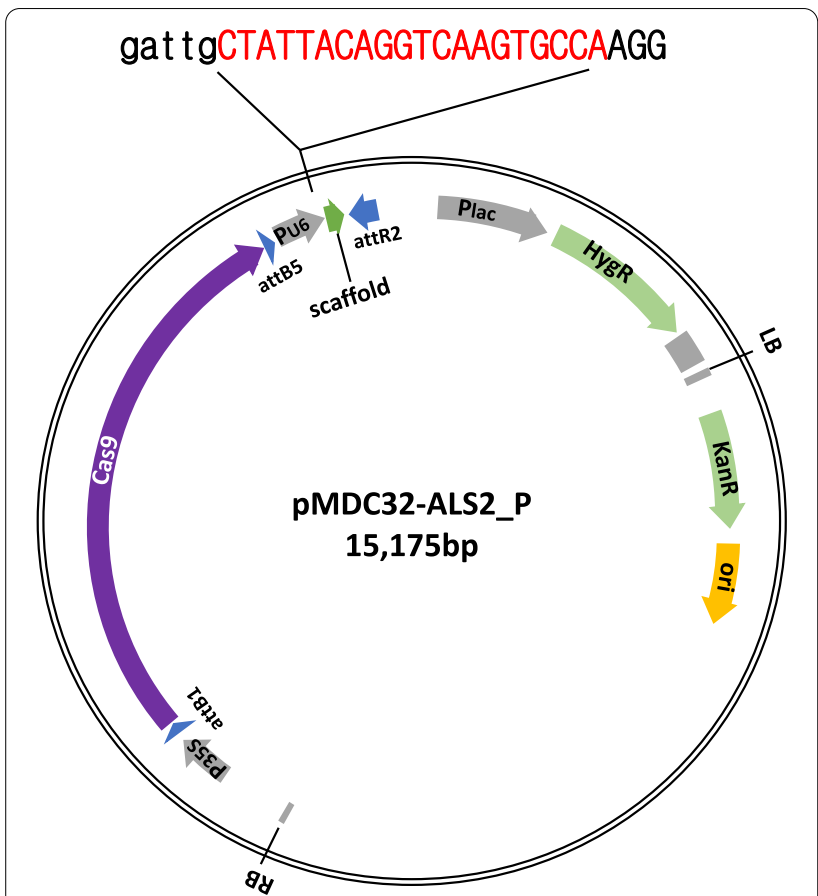

Fig. 2 Construction map used for expressing CRISPR-Cas9 system transiently. sgRNA and Cas9 are conducted with AtU6 promoter and 355 promoter, respectively. The vector contains $\mathrm{Kan}^{r}$ gene for bacterial selection and $\mathrm{Hyg}^{r}$ gene for plant selection. The ALS2_P sgRNA (the sequences in red) is indicated to present the cloning site of sgRNA

The sgRNA efficiencies of editing of the ALS genes, ALS1-W, ALS2-W and ALS2-P, were 0.4, 0.207, and 0.7, respectively (Fig. 3B). The editing efficiency of ALS2$P$ sgRNA was higher than that of the ALS2-W sgRNA, both in the transient expression test and in the analysis by the CRISPR-P program, in which the efficiency of the ALS2-P and ALS2-W were estimated to be 0.2895 and 0.0626 , respectively. This result indicates that the sgRNA efficiencies produced in this study correctly represent the values estimated by CRISPR-P 2.0. The higher values for this gene compared to the $p d s 1$ gene indicate that the ALS genes is more accessible to the CRISPR-Cas9 complex than the $p d s 1$ gene.

Finally, the tested efficiencies of the three sgRNAs targeting the EPSPS genes, EPSPS-GT, EPSPS-c1, and EPSPS-c2, were 0.35, 0.17, and 0.16, respectively (Fig. 3C). Two control sgRNAs showed limited efficiency. even though one of them, EPSPS-c1 was expected to have high editing efficiency of 0.4788 in the CRISPRCas9 system. The sgRNA for the herbicide target EPSPSGT was predicted to have effective numbers. Therefore, the accessibility of the EPSPS gene to the CRISPR-Cas9 complex varies depending on the target sequence in the EPSPS gene.
One more sgRNA for editing the tomato pectate lyase $(S l P L)$ was adopted to compare editing efficiency both in transient system and in the transgenic tomato. Designed sgRNA, SIPL_7 showed the basal editing efficiency, 0.08, which was one of the lowest efficiency of sgRNA tested in this report (Fig. 3D).

A representative base editing patterns obtained from NGS analysis for each sgRNA was shown in Fig. 4. The base editing pattern showed a typical CRISPR-Cas9 pattern (Fig. 4). Because the fourth base upstream from the PAM sequence is the target of the Cas9 endonuclease, most base deletions start from this base, and these results convinced us that the CRISPR-Cas9 system also works for transient expression. The ALS2_P sgRNA showed the highest base editing efficiency in this study, and various patterns of deletion were observed (Fig. 4B). The other sgRNAs, PDS_R, ALS1_W, ALS2_W and EPSPS_ GT, showed less variable editing patterns compared to ALS2_P (Fig. 4). Because we expected missense mutations or the target deletion of three, six, or nine bases to lead to amino acid deletion, high efficiency of the sgRNA is required. We found that the missense mutation efficiency of ALS2_P was $0.37 \%$ when the sgRNA efficiency was $1.31 \%$ (Fig. 4B). However, the missense mutation efficiency was less than $0.1 \%$ in the other sgRNAs. Therefore, we found that high sgRNA efficiency is important to obtain enough missense mutants to generate herbicideresistant crops. We tested four different herbicide target genes, and concluded that the ALS2-P sgRNA was the best target for the production of transgenic tomatoes. ALS1-W sgRNA was the second most promising candidate. The SIPL_7 sgRNA had basal editing efficiency, however, it showed the typical In/Del patterns generated by CRISPR/Cas9 system (Fig. 4D). Therefore, we used SIPL_7 sgRNA for comparing sgRNA efficiency in transgenic plants with selected sgRNAs targeting ALS genes.

\section{Generating ALS gene edited tomatoes}

Using the same vector system for transient expression, sgRNAs targeting ALS2_P and ALS1_W, and the Cas9 gene were transformed into tomato plants using Agrobacterium mediated transformation. Eleven independent ALS1_W edited tomatoes and eight independent ALS2_P edited tomato were generated. All 19 independent transgenic plant showed different base editing patterns as shown in Fig. 5. From 19 independent generated tomatoes harboring different ALS gene sequences, two had three base deletion mutations, a modification which is important to obtain missense mutants of the target gene (Fig. 5). The proportion of missense mutations in the base editing event was therefore about $10 \%$. There were no base substitutions. In case of SlPL_7 sgRNA, we generated 60 independent 
Table 2 Primer sets for amplifying target genes

\begin{tabular}{|c|c|c|c|}
\hline Target gene & Primer & & Sequence \\
\hline \multirow[t]{4}{*}{ PDS_C } & 1st primer & Forward & GAGCTCGAGGTCGTCTTCTT \\
\hline & & Reverse & TGCTCCTAGTCCAATCAGCAG \\
\hline & 2nd primer & Forward & ACACTCTTTCCCTACACGACGCTCTTCCGATCTTGGTTAAGGACTTGGGGCCT \\
\hline & & Reverse & GTGACTGGAGTTCAGACGTGTGCTCTTCCGATCTTTTAGTTGGGCGCGGAGAAG \\
\hline \multirow[t]{4}{*}{ PDS_R } & 1st primer & Forward & TGTTAAGGACTGGATGAGAAAGCA \\
\hline & & Reverse & ATATGCCCTCAACCTAGAGAAACC \\
\hline & 2nd primer & Forward & ACACTCTTTCCCTACACGACGCTCTTCCGATCTATTGCTATGTCAAAGGCACTCAAC \\
\hline & & Reverse & GTGACTGGAGTTCAGACGTGTGCTCTTCCGATCTATGTTATGTGAGAGTCTGGAAGGG \\
\hline \multirow[t]{4}{*}{ ALS1_W } & 1st primer & Forward & TGTGGCGAGTACTTTGATGGGACT \\
\hline & & Reverse & GCACCATTACTGGGAATCATAGGC \\
\hline & 2nd primer & Forward & ACACTCTTTCCCTACACGACGCTCTTCCGATCTGTAGACATCGATGGGGATGGTAGT \\
\hline & & Reverse & GTGACTGGAGTTCAGACGTGTGCTCTTCCGATCTCACAAGCCTCCGCGAATTTCAACA \\
\hline \multirow[t]{4}{*}{ ALS2_W } & 1st primer & Forward & TCCAACTGGGGATGAGCTTTCACT \\
\hline & & Reverse & GCCCTCCGTGATCACATCTTTGAA \\
\hline & 2nd primer & Forward & ACACTCTTTCCCTACACGACGCTCTTCCGATCTGTTGACATTGACGGTGATGGGAGT \\
\hline & & Reverse & GTGACTGGAGTTCAGACGTGTGCTCTTCCGATCTGAAGGGTCACCCAAGTAAGTGTGT \\
\hline \multirow[t]{4}{*}{ ALS2_P } & 1st primer & Forward & CCCATAACGACGTTTCTGAACCTG \\
\hline & & Reverse & CCCTCCAATATGGAATTCAAACCC \\
\hline & 2nd primer & Forward & ACACTCTTTCCCTACACGACGCTCTTCCGATCTGGTGTTTGCATTGCTACCTCTGGT \\
\hline & & Reverse & GTGACTGGAGTTCAGACGTGTGCTCTTCCGATCTGCTTCACGAACAACCCTAGGAATA \\
\hline \multirow[t]{4}{*}{ EPSPS_GT } & 1st primer & Forward & TGACGACATTCATTACATGCTTGG \\
\hline & & Reverse & ATGCTTTCCCAGGAGATTTGTACT \\
\hline & 2nd primer & Forward & ACACTCTTTCCCTACACGACGCTCTTCCGATCTTGACAATGAAAACCAACGAGCAAT \\
\hline & & Reverse & GTGACTGGAGTTCAGACGTGTGCTCTTCCGATCTGGTCTCTCTCTCATCCTAGGAACT \\
\hline \multirow[t]{4}{*}{ EPSPS_C1 } & 1st primer & Forward & AAGGGATACAGACCCTTAGTCTGA \\
\hline & & Reverse & GCTGCTTAAGACCATCAACCAAAT \\
\hline & 2nd primer & Forward & ACACTCTTTCCCTACACGACGCTCTTCCGATCTTTTTAGGATTTCTGCATCAGTGGC \\
\hline & & Reverse & GTGACTGGAGTTCAGACGTGTGCTCTTCCGATCTAAATTGTCAACAACAGTCCTTCCC \\
\hline \multirow[t]{4}{*}{ EPSPS_C2 } & 1st primer & Forward & TGACGACATTCATTACATGCTTGG \\
\hline & & Reverse & ATGCTTTCCCAGGAGATTTGTACT \\
\hline & 2nd primer & Forward & ACACTCTTTCCCTACACGACGCTCTTCCGATCTTGTCGGTAAAAAGTCTGAGGAAGA \\
\hline & & Reverse & GTGACTGGAGTTCAGACGTGTGCTCTTCCGATCTGCTGCTTAAGACCATCAACCAAAT \\
\hline
\end{tabular}

tomatoes targeting SIPL gene, however, none of the transgenic plants were edited by SIPL_7 sgRNA. Therefore, we confirmed that the low efficiency of sgRNA in the transient expression test, which was 0.08 in case of SIPL_7, appear to low target editing efficiency in the transgenic plant generation.

We tested the possibility of producing herbicideresistant tomatoes using the CRISPR-Cas9 system, by targeting the PDS, ALS, and EPSPS genes. We selected the best two sgRNAs for editing ALS genes, and were able to generate a small number of transgenic tomatoes that may have altered sensitivity to ALS-targeting herbicides. The chance of obtaining altered herbicide sensitivity in transgenic tomatoes was around $10 \%$ (two transgenic plant from 19 independent lines). Several base editing methods have been used for base substitution at the herbicide target site [38]. However, these base editors have lower activity than normal Cas9, or need codon optimization for expression in plants [40]. We propose that base deletions with high efficiency of sgRNA using plant codon optimized Cas9 is another option for the generation of herbicide-resistant crops. The sensitivity of the transgenic tomatoes to herbicide was not tested in this study, because there are around 60 different herbicides targeting ALS, and it is difficult to predict the most appropriate combination [41]. To demonstrate the generation of herbicide-tolerant tomatoes using the CRISPR-Cas9 system, herbicide screening should be performed in the next set of experiments. 
A

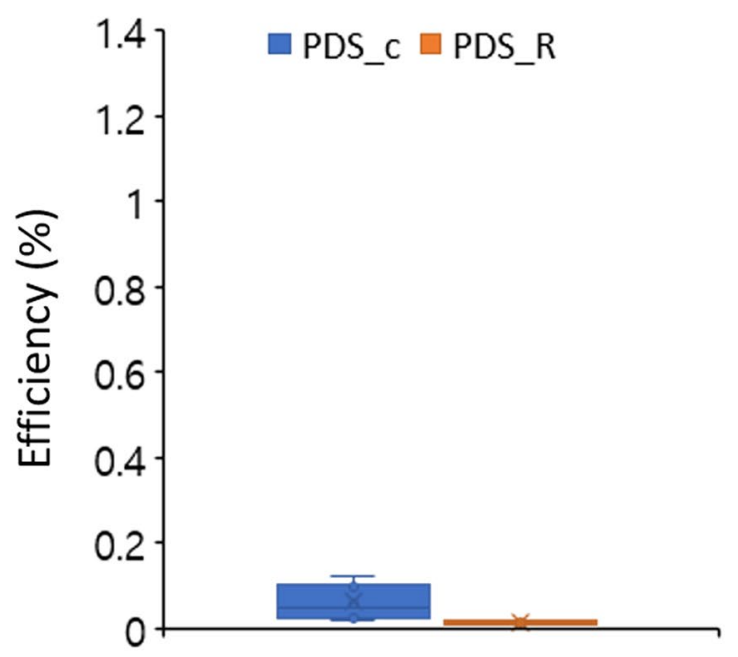

C

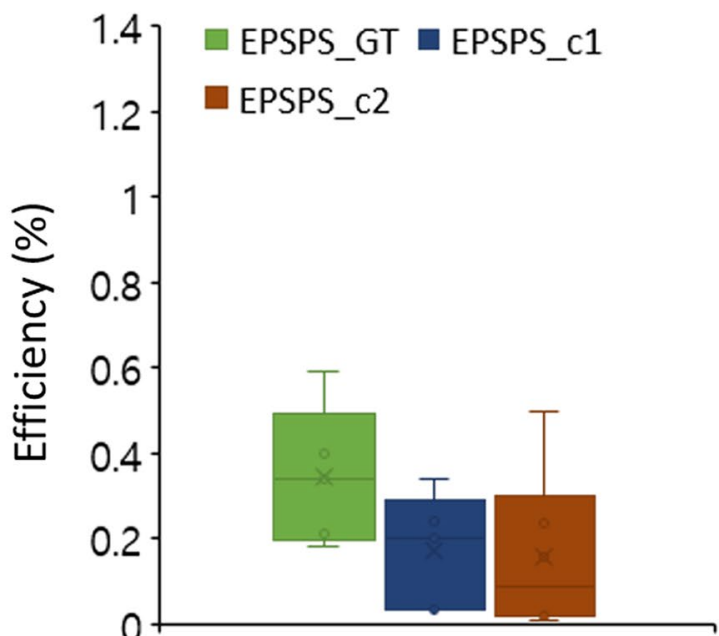

B

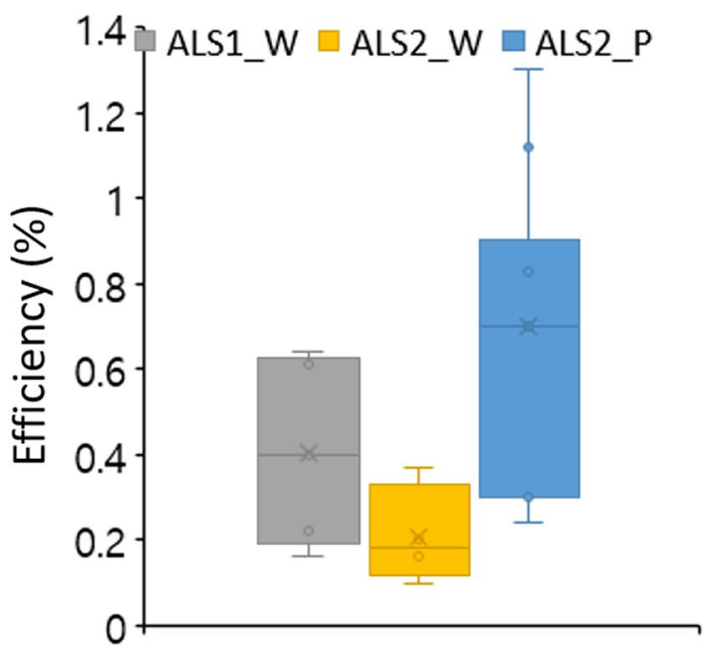

$\mathrm{D}$

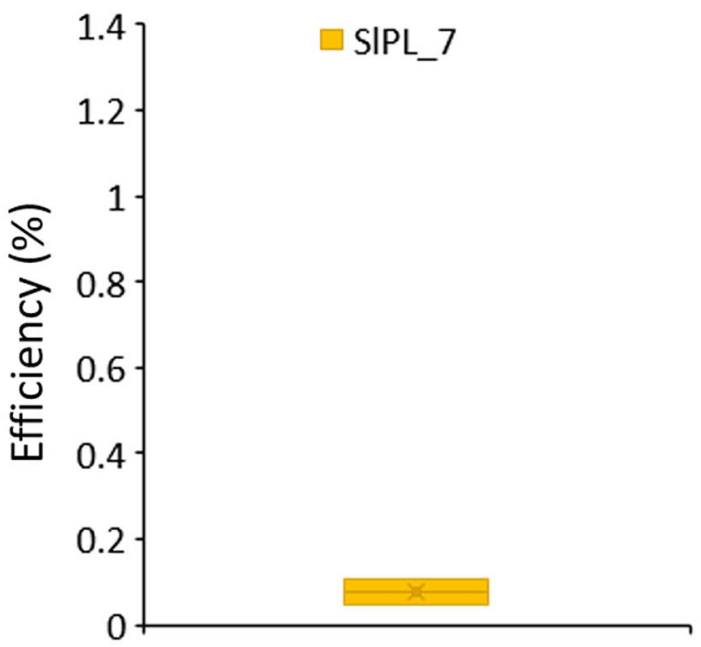

Fig. 3 sgRNA efficiency measurement. The editing efficiency of sgRNAs for three herbicide resistance gene, pds 1 (A), ALSs (B), EPSPS (C), and PL (D) are measured through Agrobacterium mediated transient expression in the tomato cotyledon. The box graph indicates Q1 (25\%), Q2 (50\%, median), and Q3 (75\%) of measured sample groups. The mean value is indicated as x mark inside of the box. The top and bottom whisker lines indicate maximum and minimum values measured, respectively. ALS2_P and ALS1_W were selected as two highly efficient sgRNAs

\section{Methods}

\section{gRNA design}

The guide RNA (gRNA) of the gene targeted for gene editing was designed using the CRISPR-P 2.0 software (http://crispr.hzau.edu.cn/cgi-bin/CRISPR2/CRISPR). Using CRISPR-P 2.0, a sequence of 20 bp in the 5' direction from the Proto-Spacer Adjacent Motif (PAM, 5'-NGG) sequence recognized by the CRISPR/Cas9 system in the target gene of the tomato genome was selected as the gRNA. We selected gRNAs predicted by the program to have high target specificity and low off-target activity. The target up was 5'-GATTGN ${ }_{20}-3^{\prime}$ and the target down was: 5'-AAACN ${ }_{20} \mathrm{C}-3$ '. Oligos were designed for two PDS genes, three ALS genes, and three EPSPS genes (Table 1). 
A

\begin{tabular}{clll}
\hline $\begin{array}{c}\text { Gene } \\
\text { name }\end{array}$ & Target sequence & \#read Effc. \\
\hline & TTAACTGTATTGTCCAGCTC 41609 & \\
& TTAACTGTATTGT-CAGCTC & 16 & \\
PDS & TTAACTGTATTGTCC-CTC & 14 & \\
_C & TTAACTGTATT-----GCTC & 4 & \\
& TTAACTGTATTGTCCAGTCTC & 3 & \\
& TTAACTGTATTGTCCAGACTC & 3 & \\
& TTAACTGTATTGTCCAGGCTC & 2 & $0.12 \%$ \\
\hline \multirow{2}{*}{ PDS } & CGCATTGAACAGGTTCTTC 33245 \\
_R & CGCATTGAACAGGTTTCTT- & 3 & \\
& CGCATT-AACAGGTTCTTC & 2 & $<0.1 \%$ \\
\hline
\end{tabular}

C

\begin{tabular}{|c|c|c|c|}
\hline $\begin{array}{l}\text { Gene } \\
\text { name }\end{array}$ & Target sequence & \#read & Effc. \\
\hline \multirow{12}{*}{$\begin{array}{c}\text { EPSPS } \\
\text { GT }\end{array}$} & TTGCTGTTCCTGCATTTCCA & 83453 & \\
\hline & TTGCTGTTCCTGCA--TCCA & 80 & \\
\hline & TTGCTGTTCCTGCA---CCA & 65 & \\
\hline & TTGCTGTTCCTGCA-TTCCA & 17 & \\
\hline & TTGCTGTTCCTGCATTT-CA & 3 & \\
\hline & TTGCTGTTCCTGCATTTCC- & 3 & \\
\hline & -TGCTGTTCCTGCATTTCCA & 3 & \\
\hline & T-CTGTTCCTGCATTTCCA & 3 & \\
\hline & TT-CTGTTCCTGCATTTCCA & 3 & \\
\hline & TTGCTG-TCCTGCATTTCCA & 2 & \\
\hline & TTGCTGTTCCTGCA---CCA & 2 & \\
\hline & TTGCTGTT-CTGCATTTCCA & 2 & $0.21 \%$ \\
\hline \multirow{3}{*}{$\begin{array}{c}\text { EPSPS } \\
\text { _c1 }\end{array}$} & TCTGGTACTGTTAAATTACC & 11419 & \\
\hline & TCTGGTACTG------TACC & 37 & \\
\hline & TCTGGTACTGTTAAATT-CC & 2 & $0.34 \%$ \\
\hline \multirow{8}{*}{$\begin{array}{c}\text { EPSPS } \\
-c 2\end{array}$} & CTGTAGCTGGAGGACATTCA & 49066 & \\
\hline & CTGTAGCTGGAGGACA-TCA & 92 & \\
\hline & CTGTAGCTGGAGGAC--TCA & 54 & \\
\hline & CTGTAGCTGGAGG--ATTCA & 52 & \\
\hline & CTGTAG---------CATTCA & 23 & \\
\hline & CTGTAGCTGGA-------CA & 18 & \\
\hline & C-TAGCTGGAGGACATTCAAGG & 2 & \\
\hline & CTGTAGCT-GAGGACATTCAAGG & 2 & $0.5 \%$ \\
\hline
\end{tabular}

B

\begin{tabular}{|c|c|c|c|}
\hline $\begin{array}{l}\text { Gene } \\
\text { name }\end{array}$ & Target sequence & \#read & Effc. \\
\hline \multirow{7}{*}{$\begin{array}{l}\text { ALS1 } \\
-W\end{array}$} & ATTTGGGAATGGCGACACAG & 57578 & \\
\hline & ATTTGGGAATGGCG--ACAG & 107 & \\
\hline & ATTTGGGAATGGCGAC-CAG & 46 & \\
\hline & ATTT-GGAATGGCGACACAG & 3 & \\
\hline & ATTTGGGAAT-GCGACACAG & 2 & \\
\hline & ATTTGGG-ATGGCGACACAG & 2 & \\
\hline & ATTTGGGAATGGCGACAACAG & 49 & $0.64 \%$ \\
\hline \multirow{8}{*}{$\begin{array}{l}\text { ALS2 } \\
\text {-W }\end{array}$} & ACTTGGGAATGGTGGTTCAA & 101013 & \\
\hline & ACTTGGGAATGGTGG-TCAA & 82 & \\
\hline & ACTTGGGAATGG----TCAA & 23 & \\
\hline & ACTTGGGAA------CCCAA & 22 & \\
\hline & ACTTGGGAATGGT-GTTCAA & 7 & \\
\hline & ACTTGGGAAT-GTGGTTCAA & 7 & \\
\hline & ACTTGGGAATG------CAA & 3 & \\
\hline & ACTTGGGAATGGTGGTTTCAA & 38 & $0.37 \%$ \\
\hline \multirow{15}{*}{$\begin{array}{c}\text { ALS2 } \\
{ }_{-}^{P}\end{array}$} & CTATTACAGGTCAAGTGCCA & 21094 & \\
\hline & CTATTACAGGTCAA---CCA & 60 & \\
\hline & CTATTACAGGTCAAGT-CCA & 48 & \\
\hline & CTATTACAGGTCA----CCA & 40 & \\
\hline & CTATTACAGGTC-----CCA & 30 & \\
\hline & CTATTACAGGT------CCA & 16 & \\
\hline & CTATTACAGGTCAA--GCCA & 11 & \\
\hline & CTATTACAGGTCA--TGCCA & 9 & \\
\hline & CTATTACAGGT-------CA & 3 & \\
\hline & CTATTACAGGTCA-----CA & 2 & \\
\hline & CTATTACAGGTCA---GCCA & 2 & \\
\hline & CTATTACAG-------GCCA & 2 & \\
\hline & CTATTACAGGTCAAGTGACCA & 15 & \\
\hline & CTATTACAGGTCAAGTGGCCA & 12 & \\
\hline & CTATTACAGGTCAAGTGTCCA & 5 & $1.31 \%$ \\
\hline \multicolumn{4}{|l|}{$D$} \\
\hline $\begin{array}{l}\text { Gene } \\
\text { name }\end{array}$ & Target sequence & \#read & Effc. \\
\hline \multirow{7}{*}{ SIPL7 } & ATGCCGTCACTCCGAAGCCG & $\begin{array}{c}11599 \\
4\end{array}$ & \\
\hline & ATGCCGTCACTCCGAA-CCG & 82 & \\
\hline & ATGCCGTCACTCCGAAGCC- & 32 & \\
\hline & ATGCCGTCACTCCGAAG-CG & 6 & \\
\hline & ATGCCGTCACT-CGAAGCCG & 3 & \\
\hline & ATG-CGTCACTCCGAAGCCG & 2 & \\
\hline & ATGCCGTCACTCCGAAGGCCG & 32 & $0.135 \%$ \\
\hline
\end{tabular}

Fig. 4 Editing patterns generated by sgRNAs in transient test. Edition of target gene pds1 (A), ALSS (B), EPSPS (C), and PL (D) is confirmed by NGS analysis. The target sequence is indicated in the first row of each section and In/Del sequences are presented in red dashes/letters. The efficiency of selected case is indicated in the right bottom line in each section (Effc.). The efficiency was calculated by dividing In/Del reads with total read 


\begin{tabular}{|c|c|}
\hline $\begin{array}{c}\text { ALS1 } \\
-W\end{array}$ & 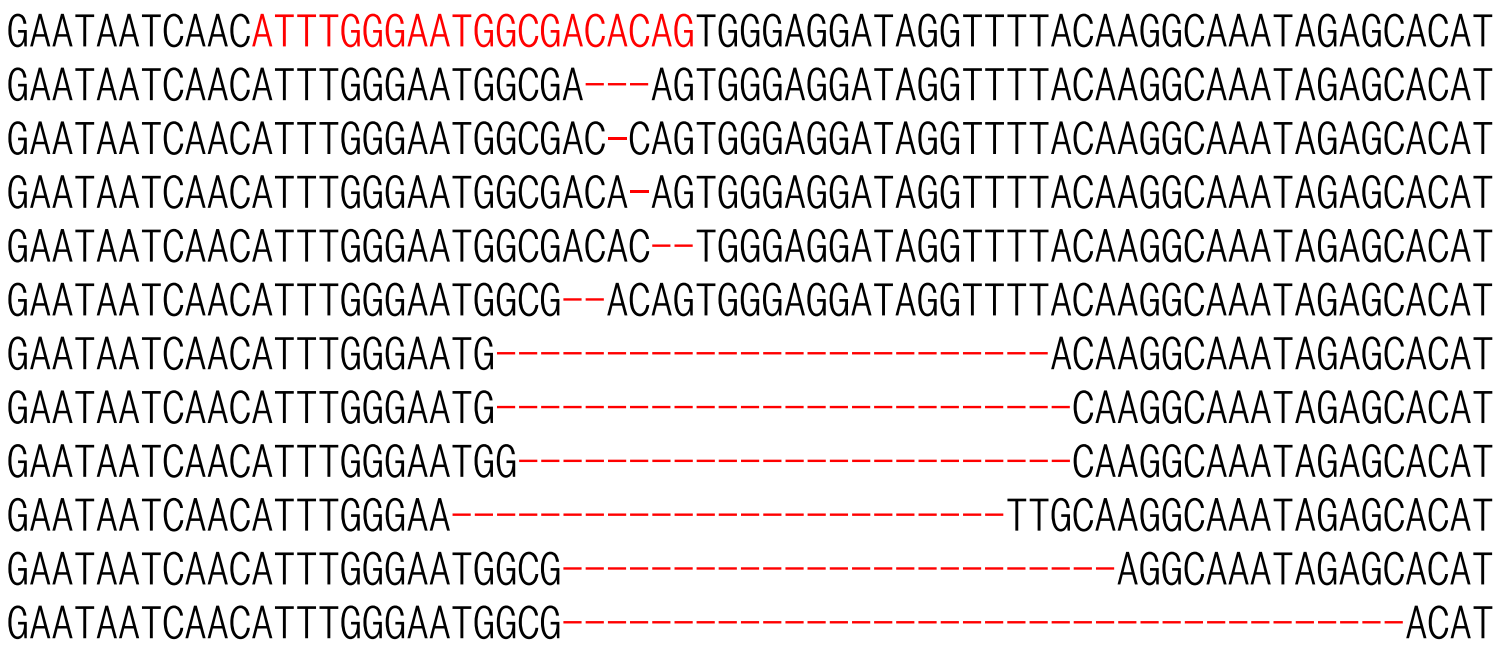 \\
\hline $\begin{array}{c}\text { ALS2 } \\
{ }_{-}^{P}\end{array}$ & 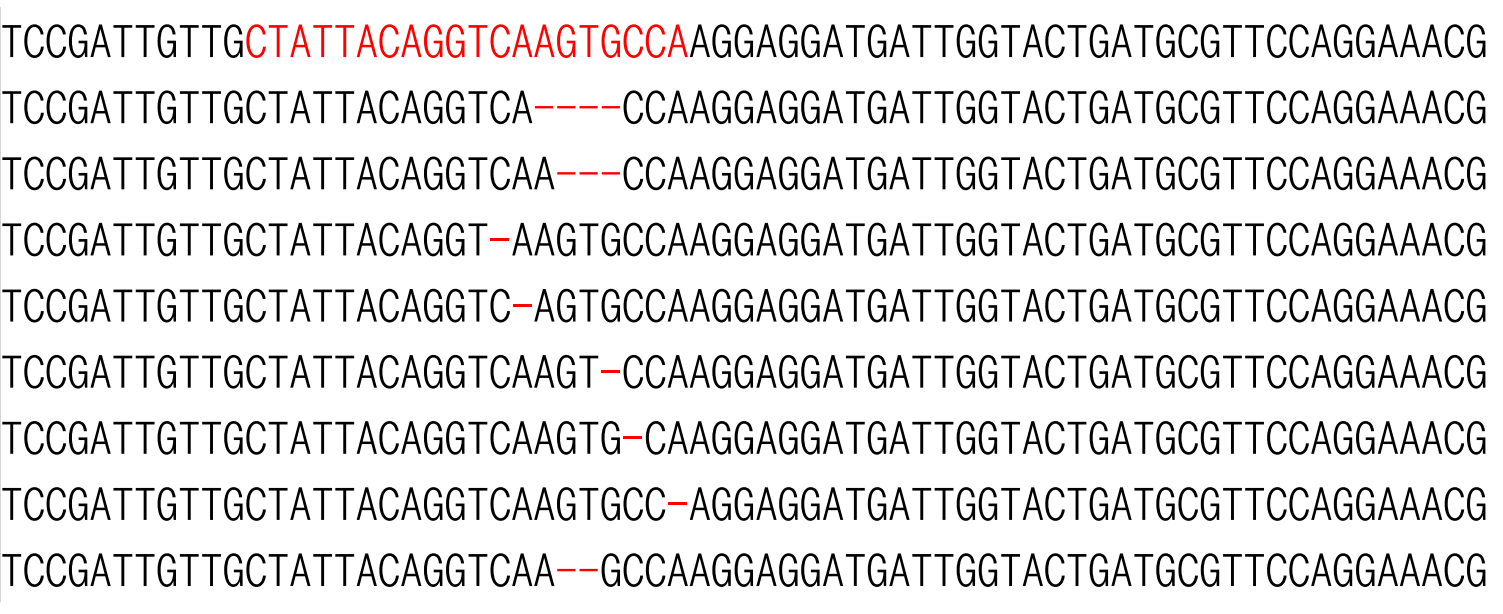 \\
\hline \multicolumn{2}{|r|}{$\begin{array}{l}\text { Fig. } 5 \text { Regeneration of transgenic tomatoes and confirming gene edition. The transgenic tomatoes were regenerated from Agrobacterium } \\
\text { mediated transformation. The sequences of ALS genes of transgenic tomatoes are presented. The target sequence is indicated in red letters and } \\
\text { deleted sequences are presented in red dashes }\end{array}$} \\
\hline
\end{tabular}

\section{Cloning using the MDC32 vector}

To transiently express or modify the Cas9 system in tomatoes, the Lowder et al. (2015) cloning system was used [39]. Annealed double-stranded gRNAs were generated by lowering the temperature by $1{ }^{\circ} \mathrm{C}$ every $5 \mathrm{~s}$ for $10 \mathrm{~min}$ at $95{ }^{\circ} \mathrm{C}$ in a total volume of $25 \mu \mathrm{l}$ of $10 \mathrm{pmol}$ of target up oligo and $10 \mathrm{pmol}$ of target down oligo for the selected gRNA. The double-stranded gRNA fragment was ligated into the PYPQ141A vector treated with the restriction enzyme Esp3I. Next, pYPQ141A-gRNA plasmid DNA, the pYPQ154 vector containing Cas9, and pMDC32, a target vector for plant transformation, were subjected to the recombination (LR) reaction to obtain a final transformation vector (Fig. 1). The cloned vector was transformed with the Agrobacterium GV3101 vector, and then used for tomato transformation or transient expression.

\section{Transient expression of CRISPR-Cas9 system in the cotyledon of tomato}

To evaluate the operation and efficiency of gRNA using the CRISPR-Cas9 system, an Agrobacterium suspension was injected into tomato cotyledons, and the efficiency of the gRNA was confirmed by calculating the 
corrected efficiency in the cotyledon genome. Agrobacterium seed cultures were cultured at $28{ }^{\circ} \mathrm{C}$ for $24 \mathrm{~h}$ in $5 \mathrm{~mL} \mathrm{LB}$ medium supplemented with $50 \mu \mathrm{g} / \mathrm{mL}$ of kanamycin, rifampicin, or gentamicin, respectively. For culturing Agrobacterium for injection, 10\% of seed cells were inoculated into $5 \mathrm{~mL}$ LB medium and then cultured at $28{ }^{\circ} \mathrm{C}$ until 0.8 (Biochrom Libra S22, Biochrom, Cambridge, UK) with A600 nm absorbance. After precipitating $1 \mathrm{~mL}$ of cultured Agrobacterium at $7000 \mathrm{rpm}$ for $1 \mathrm{~min}$, the cells were resuspended in infiltration buffer (distilled water $50 \mathrm{~mL}$, MS vitamin $0.05 \mathrm{~g}$, sucrose $0.37 \mathrm{~g}, 1 \mathrm{mM}$ IAA $12.5 \mu \mathrm{l}, 10 \mathrm{mM}$ Zeatin $12.5 \mu \mathrm{l}, 10 \mathrm{mM}$ Zeatin $12.5 \mu \mathrm{M}$ A1 $\mu$ 20) and acclimated at $28{ }^{\circ} \mathrm{C}$ for $3 \mathrm{~h}$. For infiltration, about $1 \mathrm{~mL}$ of Agrobacterium solution was evenly infiltrated throughout the leaf using a syringe with the needle removed, from the back (axial direction) of the leaf. Tomato cotyledons were grown for 10-15 d. The injected tomato cotyledons were cultured in a chamber at $24{ }^{\circ} \mathrm{C}$ for $5 \mathrm{~d}$.

\section{Preparation of genomic DNA from plant tissue and analysis of genome editing}

A rapid DNA extraction method was used for genomic DNA extraction [42]. To confirm the genotype of the target tomato gene edited by CRISPR-Cas9, the target gene was PCR-amplified from the extracted genomic DNA using the primers shown in Table 2. The DNA fragments of each gene amplified by PCR were sequenced with a read level of 20,000-100,000, using NGS. For sequencing, gene diversity analysis was performed using the CRISPR RGEN Tools (http://www. rgenome.net/cas-analyzer) [43].

\section{Abbreviations}

ALS: Acetolactate synthase; CRISPR: Clustered regular interspaced short palindromic repeats; EPSPS: 5-Enolpyruvylshikimate-3-phosphate synthase; sgRNA: Single guide RNA; NGS: Next-generation sequencing; PAM: Proto-Spacer Adjacent Motif; pds: Phytoene desaturase; TALENs: Transcription Activator-Like Effector Nucleases.

\section{Acknowledgements}

Not applicable.

\section{Authors' contributions}

Conducting experiment, SHY, EK, HP; Writing, SHY, YK; Investigation, YK. All authors read and approved the final manuscript.

\section{Funding}

Y.K. received funding from "Cooperative Research Program for Agriculture Science and Technology Development" of Rural Development Administration of Korea (Project No. PJ01477601), and from by the National Research Foundation of Korea (No. NRF-2020R1F1A1075575).

\section{Availability of data and materials}

The datasets used and/or analyzed during the current study are available from the corresponding author on reasonable request.

\section{Declarations}

\section{Competing interests}

The authors declare that they have no competing interests.

Received: 10 November 2021 Accepted: 18 January 2022

Published online: 09 February 2022

\section{References}

1. Gressel J (2009) Evolving understanding of the evolution of herbicide resistance. Pest Manag Sci 65:1164-1173

2. Busi R, Vila-Aiub MM, Beckie HJ, Gaines TA, Goggin DE, Kaundun SS, Lacoste M, Neve P, Nissen SJ, Norsworthy JK, Renton M, Shaner DL, Tranel PJ, Wright T, Yu Q, Powles SB (2013) Herbicide-resistant weeds: from research and knowledge to future needs. Evol Appl 6:1218-1221

3. Sundstrom J, Albihn A, Boqvist S, Ljungvall K, Marstorp H, Martiin C, Nyberg K, Vagsholm I, Yuen J, Magnusson U (2014) Future threats to agricultural food production posed by environmental degradation, climate change, and animal and plant diseases - a risk analysis in three economic and climate settings. Food Secur 6:201-215

4. Heap I (2014) Global perspective of herbicide-resistant weeds. Pest Manag Sci 70:1306-1315

5. Green JM (2014) Current state of herbicides in herbicide-resistant crops. Pest Manag Sci 70:1351-1357

6. Gage KL, Krausz RF, Walters SA (2019) Emerging challenges for weed management in herbicide-resistant crops. Agriculture 9:180

7. Green JM, Owen MD (2011) Herbicide-resistant crops: utilities and limitations for herbicide-resistant weed management. J Agric Food Chem 59:5819-5829

8. Yi SY, Cui Y, Zhao Y, Liu ZD, Lin YJ, Zhou F (2016) A novel naturally occurring class i 5-enolpyruvylshikimate-3-phosphate synthase from Janibacter sp. confers high glyphosate tolerance to rice. Sci Rep 6:19104

9. Leino L, Tall T, Helander M, Saloniemi I, Saikkonen K, Ruuskanen S, Puigbo P (2021) Classification of the glyphosate target enzyme (5-enolpyruvylshikimate-3-phosphate synthase) for assessing sensitivity of organisms to the herbicide. J Hazard Mater 408:124556

10. Heap I, Duke SO (2018) Overview of glyphosate-resistant weeds worldwide. Pest Manag Sci 74:1040-1049

11. Jakeman DL, Mitchell DJ, Shuttleworth WA, Evans JNS (1998) On the mechanism of 5-enolpyruvylshikimate-3-phosphate synthase. Biochemistry-Us 37:12012-12019

12. Liu Y, Cao G, Chen R, Zhang S, Ren Y, Lu W, Wang J, Wang G (2015) Transgenic tobacco simultaneously overexpressing glyphosate $\mathrm{N}$-acetyltransferase and 5-enolpyruvylshikimate-3-phosphate synthase are more resistant to glyphosate than those containing one gene. Transgenic Res 24:753-763

13. Le DT, Choi JD, Tran LS (2010) Amino acids conferring herbicide resistance in tobacco acetohydroxyacid synthase. GM Crops 1:62-67

14. Yu Q, Han H, Vila-Aiub MM, Powles SB (2010) AHAS herbicide resistance endowing mutations: effect on AHAS functionality and plant growth. J Exp Bot 61:3925-3934

15. Yu Q, Powles SB (2014) Resistance to AHAS inhibitor herbicides: current understanding. Pest Manag Sci 70:1340-1350

16. Sato H, Shimizu T, Kawai K, Kaku K, Arakawa A, Tachibana T, Takamizo T (2013) Herbicide-resistant tall fescue with cytoplasmic male sterility selected by a mutated rice acetolactate synthase gene. Crop Sci 53:201-207

17. Naing AH, Kyu SY, Pe PPW, Park KI, Lee JM, Lim KB, Kim CK (2019) Silencing of the phytoene desaturase (PDS) gene affects the expression of fruitripening genes in tomatoes. Plant Methods 15:110

18. Brausemann A, Gemmecker S, Koschmieder J, Ghisla S, Beyer P, Einsle O (2017) Structure of phytoene desaturase provides insights into herbicide binding and reaction mechanisms involved in carotene desaturation. Structure 25:1222-1232

19. Depka B, Jahns P, Trebst A (1998) Beta-carotene to zeaxanthin conversion in the rapid turnover of the D1 protein of photosystem II. FEBS Lett 424:267-270 
20. Koschmieder J, Fehling-Kaschek M, Schaub P, Ghisla S, Brausemann A, Timmer J, Beyer P (2017) Plant-type phytoene desaturase: functional evaluation of structural implications. PLoS ONE 12:e0187628

21. Arias RS, Dayan FE, Michel A, Howell J, Scheffler BE (2006) Characterization of a higher plant herbicide-resistant phytoene desaturase and its use as a selectable marker. Plant Biotechnol J 4:263-273

22. Heap IM (1997) The occurrence of herbicide-resistant weeds worldwide. Pestic Sci 51:235-243

23. Kumar V, Bellinder RR, Gupta RK, Malik RK, Brainard DC (2008) Role of herbicide-resistant rice in promoting resource conservation technologies in rice-wheat cropping systems of India: a review. Crop Prot 27:290-301

24. Ogawa T, Kawahigashi H, Toki S, Handa H (2008) Efficient transformation of wheat by using a mutated rice acetolactate synthase gene as a selectable marker. Plant Cell Rep 2:1325-1331

25. Endo M, Toki S (2013) Creation of herbicide-tolerant crops by gene targeting. J Pestic Sci 38:49-59

26. Shaner DL (2000) The impact of glyphosate-tolerant crops on the use of other herbicides and on resistance management. Pest Manag Sci $56: 320-326$

27. Mannerlof M, Tuvesson S, Steen P, Tenning P (1997) Transgenic sugar beet tolerant to glyphosate. Euphytica 94:83-91

28. Castle LA, SiehI DL, Gorton R, Patten PA, Chen YH, Bertain S, Cho HJ, Duck N, Wong J, Liu DL, Lassner MW (2004) Discovery and directed evolution of a glyphosate tolerance gene. Science 304:1151-1154

29. Adli M (2018) The CRISPR tool kit for genome editing and beyond. Nat Commun 9:1911

30. Nishihara M, Higuchi A, Watanabe A, Tasaki K (2018) Application of the CRISPR/Cas9 system for modification of flower color in Torenia fournieri. BMC Plant Biol 18:331

31. Lone BA, Karna SKL, Ahmad F, Shahi N, Pokharel YR (2018) CRISPR/ Cas9 system: a bacterial tailor for genomic engineering. Genet Res Int 2018:3797214

32. Ma X, Zhu Q, Chen Y, Liu YG (2016) CRISPR/Cas9 platforms for genome editing in plants: developments and applications. Mol Plant 29:961-974

33. Farooq R, Hussain K, Nazir S, Javed MR, Masood N (2018) CRISPR/Cas9; A robust technology for producing genetically engineered plants. Cell Mol Biol 64:31-38

34. Ran FA, Hsu PD, Wright J, Agarwala V, Scott DA, Zhang F (2013) Genome engineering using the CRISPR-Cas9 system. Nat Protoc 8:2281-2308

35. Samanta MK, Dey A, Gayen S (2016) CRISPR/Cas9: an advanced tool for editing plant genomes. Transgenic Res 25:561-573

36. Wang Y, Geng L, Yuan M, Wei J, Jin C, Li M, Yu K, Zhang Y, Jin H, Wang E, Chai Z, Fu X, Li X (2017) Deletion of a target gene in Indica rice via CRISPR/Cas9. Plant Cell Rep 36:1333-1343

37. Toda E, Okamoto T (2020) CRISPR/Cas9-based genome editing using rice zygotes. Curr Protoc Plant Biol 5:e20111

38. Dong H, Huang Y, Wang K (2021) The development of herbicide resistance crop plants using CRISPR/Cas9-mediated gene editing. Genes 12:912

39. Lowder LG, Zhang D, Baltes NJ, Paul JW 3rd, Tang X, Zheng X, Voytas DF, Hsieh TF, Zhang Y, Qi Y (2015) A CRISPR/Cas9 toolbox for multiplexed plant genome editing and transcriptional regulation. Plant Physiol 169:971-985

40. Li C, Zhang R, Meng XB, Chen S, Zong Y, Lu CJ, Qiu JL, Chen YH, Li JY, Gao CX (2020) Targeted, random mutagenesis of plant genes with dual cytosine and adenine base editors. Nat Biotechnol 38:875-882

41. van der Vyver C, Conradie T, Kossmann J, Lloyd J (2013) In vitro selection of transgenic sugarcane callus utilizing a plant gene encoding a mutant form of acetolactate synthase. In Vitro Cell Dev-PI 49(2):198-206

42. Mavrodiev EV, Dervinis C, Whitten WM, Gitzendanner MA, Kirst M, Kim S, Kinser TJ, Soltis PS, Soltis DE (2021) A new, simple, highly scalable, and efficient protocol for genomic DNA extraction from diverse plant taxa. Appl Plant Sci 9:e11413

43. Park J, Lim K, Kim JS, Bae S (2017) Cas-analyzer: an online tool for assessing genome editing results using NGS data. Bioinformatics 33:286-288

\section{Publisher's Note}

Springer Nature remains neutral with regard to jurisdictional claims in published maps and institutional affiliations.

\section{Submit your manuscript to a SpringerOpen ${ }^{\circ}$ journal and benefit from:}

- Convenient online submission

- Rigorous peer review

- Open access: articles freely available online

- High visibility within the field

- Retaining the copyright to your article

Submit your next manuscript at $\boldsymbol{\nabla}$ springeropen.com 